\title{
XXXV. Electromagnets.-V. The law of similar electromagnets
}

\section{R.H.M. Bosanquet}

To cite this article: R.H.M. Bosanquet (1886) XXXV. Electromagnets. -V. The law of similar electromagnets, Philosophical Magazine Series 5, 22:136, 298-309, DOI: $10.1080 / 14786448608627933$

To link to this article: http://dx.doi.org/10.1080/14786448608627933

册 Published online: 29 Apr 2009.

Submit your article to this journal $[\pi$

Џ Article views: 2

Q View related articles $\asymp$ 
which is more correct than the formula usually given hitherto for the ratio of the shunt- and series-windings, and which assumes absence of saturation terms.

The simplicity of these results, no less than that of the processes by which they are derived, lends additional value to the new formula of Dr. Frölich, whose work deserves to be more widely known and recognized than it now is.

City and Guilds of London Technical College, Finsbury, June 1886.

XXXV. Electromagnets.-V. The Law of similar Electromagnets. Saturation, \&c. By R. H. M. BosanqueT, St. Tohn's College, Oxford.

To the Editors of the Philosophical Magazine and Journal. GFitrlemen,

TN previous papers on Electromagnets I have (1)* indicated 1 the general nature of a formula for the moment and induction in electromagnets, and in subsequent papers $\dagger$ given the details of the complete examination of the permeabilities of many specimens of iron and steel, together with an attempt at a molecular formula for these permeabilities worked out in some detail. In the present communication I propose to give as shortly as possible an abstract of the results of a great number of experiments having special reference to the magnetic resistance of cylindrical bars of length equal to twenty times their diameter, with and without pole-pieces.

The datum in question (magnetic resistance) is that needed to define the magnetism under given electromagnetic excitation.

The experiments cover the whole region from small magnetic inductions up to saturation, or what would be commonly called so.

The experiments have been made on bars of different sizes of the proportions in question, so as to furnish for the first time an experimental examination of the law of the magnetism of similar solids. It appears to be of great interest to ascertain how far this law can be depended upon in practice.

The result is that, while in the main the law is conformed to, the irregularity shown by different specimens, especially in the extreme regions of small inductions and saturation, is very great. General deductions therefore, such as have been recently published, depending on the behaviour of single

* Phil. Mag. xvii. p. $531 . \quad$ † Ibid. xix. pp. 73, 333; xx. p. 318. 
specimens, cannot be accepted as having more demonstrative force than belongs to preliminary investigations. So far as work of this description goes, my own paper in Phil. Mag. June 1884 (xvii.) p. 531, showed in detail the properties of bars; and specially pointed out that the course of the values did not at all indicate any definite limit to the magnetism, and that the existing idea of saturation was a complete mistake (p. 535). This is now being published as a new discovery.

In subsequent papers I have shown that rings do not exhibit the same behaviour; and though some ring specimens admit of the magnetism being forced up very high, yet others do not.

Further, where the magnetism is forced up very high in rings, the whole magnetization function appears to change under the influence; and the values thus obtained are not generally capable of being satisfied by the same expression, which represents the behaviour of the specimen under inductions less than $\mathbf{B} \mathbf{b}=18,000$.

The difference in this respect between rings and bars is already indicated in my paper, Phil. Mag. 1885 (xix.) p. 79 ; and it is there pointed out that the cause is probably the difference in distribution, $i$. e. that in bars the lines of force are crowded closely only at the equatorial section.

The law of similar solids is easily deduced from the fact that magnetic resistance (quotient of potential by induction) is of linear dimensions. Hence, in similar electromagnets, where the inductions are the same, the number of current turns required is proportional to the linear dimension*.

The present two series of experiments were made on cylindrical bars having the following dimensions:-The pole-pieces of the second set were circular, had a diameter five times that of the bar, and thickness equal to the diameter of the bar. The object of the experiments on the bars with pole-pieces was mainly to obtain numbers which might assist to serve as bases for a knowledge of the behaviour of the field-magnets of dynamos.

* I have long been under the impression that this law was enunciated in substance by Sir W. Thomson. But after carefully going through the reprinted papers, the nearest I could find to it is at p. 435 ("Electrostatics and Magnetism'). But this statement does not include the proposition that the number of current-turns is proportional to the linear dimensions. And I am therefore doubtful whether the law is really due to Sir W. Thomson. 
The linear scale is varied in proportions up to $1: 5$. The largest of the masses weighed considerably over a cwt., and taxed our resources to handle it.

Dimensions of Bars.

\begin{tabular}{|c|c|c|c|c|c|c|c|}
\hline \multicolumn{4}{|c|}{ Plain Bars. } & \multicolumn{4}{|c|}{ BaRs With solid P.P. (pole-pieces). } \\
\hline Bare. & $\begin{array}{l}\text { Length, } \\
\text { centim. }\end{array}$ & $\begin{array}{l}\text { Radius, } \\
\text { centim. }\end{array}$ & $n$ coils. & Ba rs. & $\begin{array}{l}\text { Length, } \\
\text { centim. }\end{array}$ & $\begin{array}{l}\text { Radius, } \\
\text { centim. }\end{array}$ & $n$ coils. \\
\hline I. & 20 & 0.5 & 1092 & I. & $20 \cdot 6$ & 0.5 & 1121 \\
\hline II. & 20 & 0.5 & 1109 & II. & $20 \cdot 7$ & 0.5 & 1110 \\
\hline IV. & 40 & $1 \cdot 0$ & 1933 & III. & $38 \cdot 08$ & 1.0 & 2024 \\
\hline V. & 40 & 10 & 1931 & VI. & 38.08 & 10 & 2134 \\
\hline VII. & 60 & $1 \cdot 5$ & 1951 & XVII. & $57 \cdot 5$ & $1 \cdot 5$ & 2837 \\
\hline VIII. & 60 & 1.5 & 1909 & XVIII. & 57.0 & $1 \cdot 5$ & 2813 \\
\hline IX. & 80 & 20 & 2557 & $\mathrm{XV}$ & 739 & 2.04 & 3546 \\
\hline$x$. & 80 & $2 \cdot 0$ & 2543 & XVI. & $73 \cdot 9$ & 2.04 & 3574 \\
\hline XI. & 100 & 25 & 3187 & XIII. & 94.85 & 27025 & 3041 \\
\hline XII. & 100 & 25 & 3172 & XIV. & 948 & $2 \cdot 525$ & 3028 \\
\hline
\end{tabular}

The determinations of bar II. with pole-pieces were puzzling and anomalous, and were excluded. Determinations for small inductions were in all cases made in the first instance by reversing the effect of the horizontal component of the earth's magnetism. The bars were then wound uniformly from end to end, and the inductions determined by the effect on coils placed equatorially.

In many cases great discrepancies occurred even between bars of the same measurement (they were all in pairs). In these cases the determinations were repeated, and in several cases the whole of the magnetizing coils were rewound.

The results were then plotted on a large figure for each bar, and a curve was drawn by hand to represent the values as well as possible; $2 \mathrm{~B}$ was abscissa, and $\rho$ (magnetic resistance) ordinate.

It was at once evident that in all cases, almost without exception, the curve did not show $\rho=\infty$ for any finite value of 23, but rather indicated the existence of an asymptote inclined at a moderate angle to the axis of $\rho$.

The values of $\rho$ corresponding to $\mathfrak{B}=0,1000,2000$, \&c. were then measured off, and all divided by the lengths of the bars, so as to reduce them to data for bars 1 centim. long, according to the above law of similarity.

These values, with the resulting means, form the two following Tables. 
Mr. R. H. M. Bosanquet on Electromagnets.

301

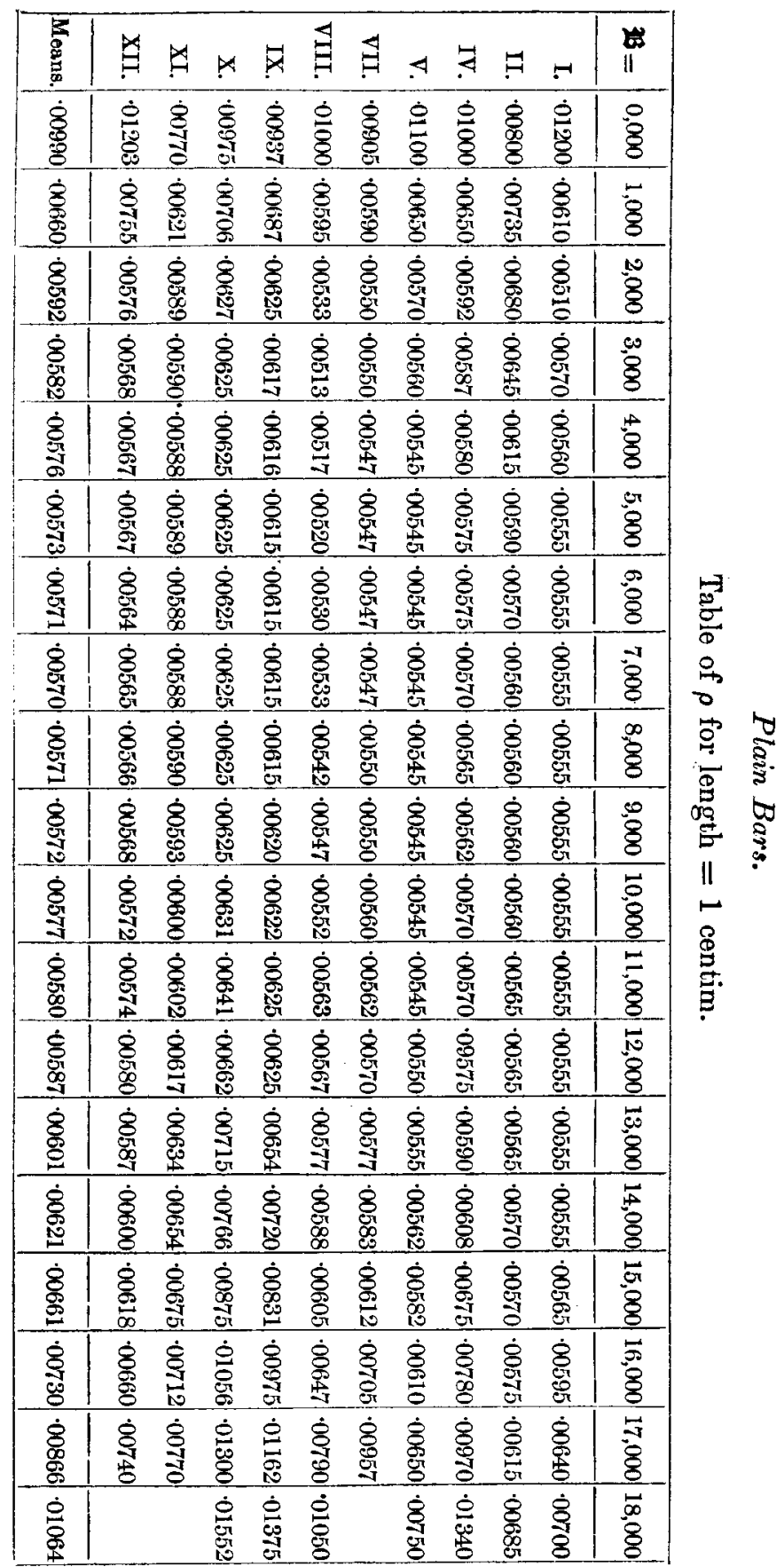




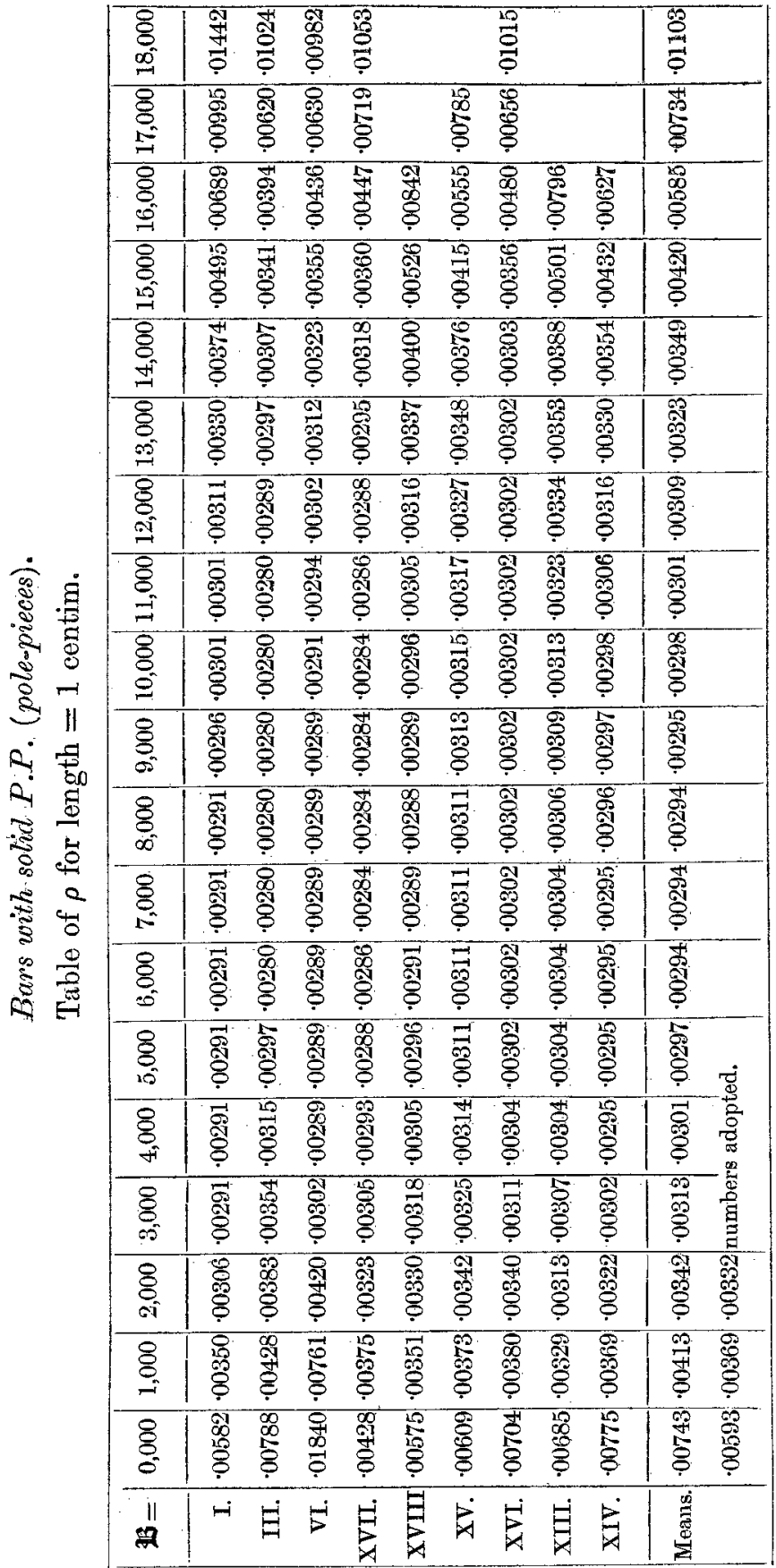


Mr. R. H. M. Bosanquet on Electromagnets.

It will be noticed that the numbers of windings are not exactly proportional to the linear dimensions. It was not practicable to carry this out; and of course it does not matter whether a given number of current-turns is made up one way or the other (by current or turns).

In the first three entries of the bars with pole-pieces, the initial discrepancies being very great, the means of selected values were adopted.

The discrepancies between the numbers in any one column are those in which any deviation from the law of similars would appear. While the values (particularly at beginning and end) are capricious in the extreme, it is certainly not possible to detect any systematic difference between the numbers belonging to the large and small bars.

Before proceeding to calculate with these numbers, I will now exhibit in a small figure the nature of the relation between the magnetic resistances due to

(1) the permeability of the metal, as derived from rings ;

(2) the bars with pole-pieces (P.P.); and

(3) plain eylindrical bars.

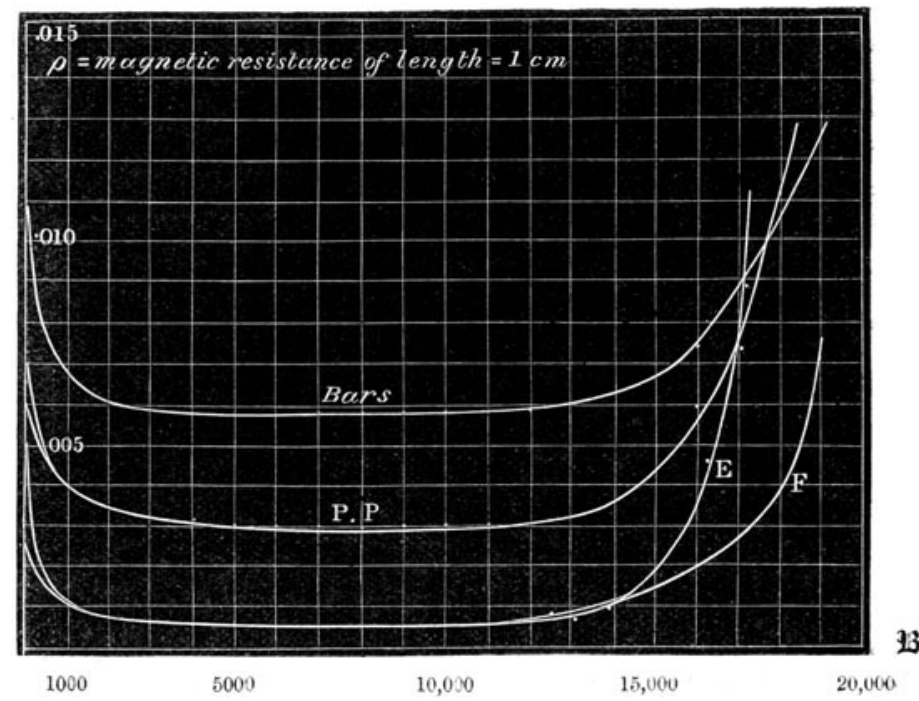

The resistance in each case is supposed to be due to a length of metal $=1$ centim., and $\rho$ is expressed in decimals of a centimetre, though drawn to a different scale for convenience. The lowest curve is made up of the values from rings $\mathrm{E}, \mathrm{F}$ 
(Phil. Mag. xix. p. 76), so as to show the wide limits within which specimens vary. The middle curve is that of the bars with pole-pieces. The initial mean and adopted value are both traced. The upper curve is that of the plain bars.

This figure illustrates, and indeed proves, for approximate purposes the rule I enunciated (Phil..Mag. xvii. p. 534), and shows how the rule includes bars with pole-pieces, and where it fails, viz. in the region spoken of as in the neighbourhood of saturation. The rule is :- " The magnetic resistance of any bar can be expressed as the sum of a resistance due to its form, and a quantity formed by dividing the length by $\mu$, the permeability of the metal."

In the figure the lowest curve represents the resistance due to the imperfect permeability of the metal. The two upper curves obviously admit of derivation from the lower one by addition of constants due to the respective shapes of the bars, allowing for the capriciousness of the values in different specimens, and for the difference in the region of saturation ( $\mathbf{B}=18,000$ and thereabouts).

As to the amount:- The analogy of the resistance to a fluid flowing from the end of a pipe would give $2 \times \cdot 6$ of the radius, or in the present case :012 centim., for the shape-resistance. The fact that magnetism issues all along the bar, more or less, diminishes this resistance; so that we find in fact that in the plain bar the addition for shape is somewhat over 005 centim., as we can see in the figure.

Again, the pole-pieces still further facilitate the flux of the magnetic induction through the ends, and the shape-resistance in this case is somewhat over 002 centim.

I proceed to a more detailed examination of the numbers.

For the plain bars, the shape-resistance calculated from the formula at Phil. Mag. xvii. p. 534 exactly satisfies the requirements of the numbers. Hence the first step is to subtract the value thus calculated from the number to be dealt with; so that $\cdot 37 r 10^{-\cdot 0061 \frac{l}{r}}=\cdot 00528$ is subtracted, and $\rho-00528=\frac{1}{\mu}$ gives the calculated values of $\mu$. These are then fitted to formulæ in accordance with the theory of Phil. Mag. xix. p. 92. With one exception, which is as follows:-Since the saturation-curve approximates to an inclined asymptote, it is impossible that can have finite maximum values; and the best way of making allowance for this has appeared to be, to substitute for the definite maximum $\mathbf{B}_{\infty}$, a sum made up of a constant $\mathrm{B}$ and a term proportional to the magnetizing 
Mr. R. H. M. Bosanquet on Electromagnets.

force, whose coefficient was determined from the observed values.

In the figure which follows, the crosses show the observed values; the dotted line shows the best representation by means of a maximum value of the induction $\mathbf{B}_{\infty}$, fitted to the highest value; and the dashed line shows the representation by means of the constant $B$ and the term proportional to the magnetizing force.

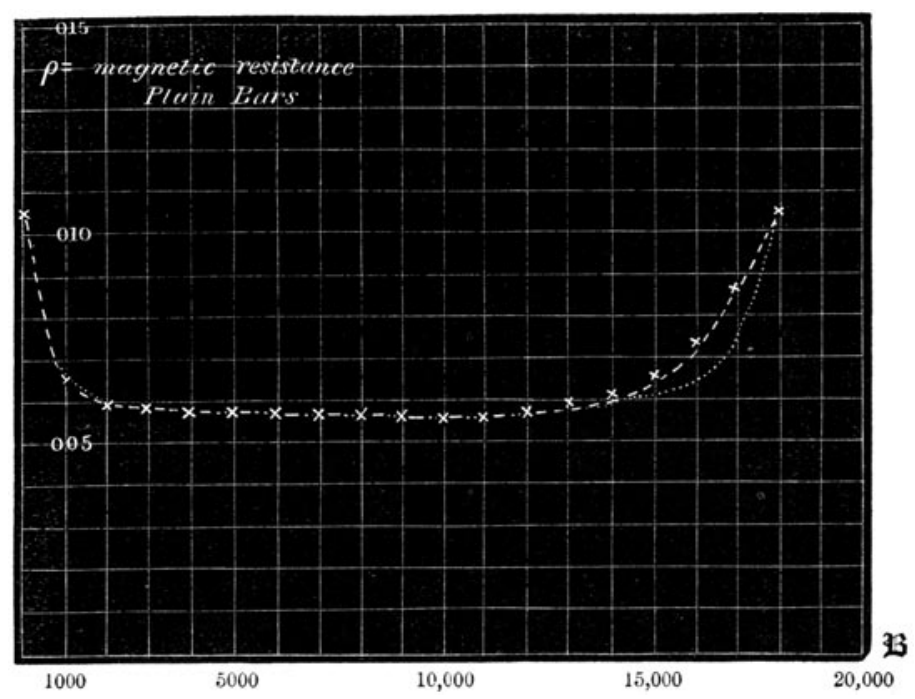

The two following Tables give the complete numerical results of the representation of the numbers for plain bars ; first, according to the calculation with a fixed maximum induction, and, secondly, where the maximum induction is made to contain a term proportional to the magnetizing force. The extraordinary gain in the representation of the saturation region in the latter case will be noticed.

$\frac{\mathbb{B}}{\mu}$ is the magnetizing force. Its coefficient $m$ in the second table is 18.5 nearly. 
Plain Bars withont Magnetizing-Force term. $\rho$ calc, $=00528+\frac{l}{\mu} \quad l=1$ centim., $\quad \log \frac{\kappa}{\rho}=2 \cdot 34170$, $\mu=395(18593-1) \cos \delta, \quad \log f=\cdot 16896$.

$$
i=f \theta, \quad \boldsymbol{i}=\frac{\kappa}{\rho} \quad \frac{60^{\circ}-\omega \theta}{\sin \theta} .
$$

\begin{tabular}{|c|c|c|c|c|c|c|}
\hline \multirow{2}{*}{23.} & \multicolumn{2}{|c|}{$\mu}$. & \multirow{2}{*}{ Diffs. } & \multicolumn{2}{|c|}{$\rho}$. & \multirow{2}{*}{ Diffs. } \\
\hline & Cale. & Obs. & & Calc. & Obs. & \\
\hline 0,000 & 188 & 188 & 0 & -01060 & .01061 & $-\cdot 00001$ \\
\hline 1,000 & 845 & 806 & +39 & .00646 & .00652 & -00006 \\
\hline 2,000 & 1366 & 1562 & -196 & .00601 & .00592 & +00009 \\
\hline 3,000 & 1766 & 1852 & -86 & .00585 & .00582 & +00003 \\
\hline 4,000 & 2052 & 2083 & -31 & .00577 & .00576 & +00001 \\
\hline 5,000 & 2239 & 2222 & +17 & -00573 & .00573 & .00000 \\
\hline 6,000 & 2343 & 2326 & +17 & $\cdot 00571$ & .00571 & .00000 \\
\hline 7,000 & 2376 & 2381 & & .00570 & .00570 & .00000 \\
\hline 8,000 & $23 \pm 6$ & 2326 & +20 & .00571 & .00571 & .00000 \\
\hline 9,000 & 2264 & 2273 & -9 & $\cdot 00572$ & .00572 & .00000 \\
\hline 10,000 & 2139 & 2041 & +98 & .00575 & $.0057 ?$ & -00002 \\
\hline 11,000 & 1979 & 1923 & +56 & .00579 & .00580 & -00001 \\
\hline 12,000 & 1785 & 1695 & +90 & .00581 & .00587 & -00003 \\
\hline 13,000 & 1566 & 1370 & +196 & .00592 & .00601 & -.00009 \\
\hline 14,000 & 1324 & 1075 & +249 & .00604 & 00521 & -00017 \\
\hline 15,000 & 1062 & 752 & +310 & .00622 & 00661 & -.00039 \\
\hline 16,000 & 784 & 495 & +289 & $\cdot 00656$ & .00730 & -00074 \\
\hline $17,0+0)$ & 491 & 296 & +195 & .00732 & .00866 & -00134 \\
\hline 18,000 & $186^{\circ}$ & 186 & 0 & .01065 & 01064 & +00001 \\
\hline
\end{tabular}

Plain Bars with Magnetizing-Force term.

$\rho$ calc. $=00528+\frac{l}{\mu}, \quad l=1$ centim.,$\quad \log \frac{\kappa}{\rho}=254133$,

$$
\begin{gathered}
\mu=65\left(\frac{n}{\mu}+16,640-25\right) \cos \delta, \quad \log f=17126, \\
\delta=f \theta, \quad \boldsymbol{B}=\frac{\kappa}{\rho} \quad \cdots 0^{2}+\omega \\
\sin \theta
\end{gathered}
$$

\begin{tabular}{|c|c|c|c|c|c|c|}
\hline \multirow{2}{*}{ B. } & \multicolumn{2}{|c|}{$\mu$. } & \multirow{2}{*}{ Diffs. } & \multicolumn{2}{|c|}{$\rho}$. & \multirow{2}{*}{ Difl's. } \\
\hline & Calc. & Obs. & & Culc. & Obs. & \\
\hline 0,000 & 189 & 188 & +1 & $\cdot 01057$ & 01061 & -00004 \\
\hline 1,000 & 813 & & & 00651 & .00652 & -00001 \\
\hline 2,000 & 1326 & 1562 & -236 & 00603 & .00502 & +000111 \\
\hline 3,000 & 1731 & 1852 & -121 & .00586 & $\cdot 00582$ & $+\cdot 00004$ \\
\hline 4,000 & 2028 & 2083 & $-5 \tilde{3}$ & .00577 & 00576 & +.001001 \\
\hline 5,000 & 2235 & 2222 & +13 & .60573 & 00573 & .00000 \\
\hline 6,000 & 2355 & 2326 & +29 & .00570 & 00571 & -06001 \\
\hline 7,0000 & 2394 & 0381 & +13 & 60570 & 00570 & $0+0 k 0$ \\
\hline 8,600 & 2391 & 2326 & +65 & 00570 & 00571 & $-000(1) 1$ \\
\hline 9,000 & 2271 & 2273 & -2 & 00572 & 60172 & .00000 \\
\hline 10,000 & 2123 & 2041 & +82 & 00575 & 00675 & -00002 \\
\hline 11,000 & 1924 & 1923 & +1 & .00580 & .00580 & .00000 \\
\hline 12,000 & 1683 & 1605 & -12 & (1)058i & $(10) \div 87$ & .00000 \\
\hline 13,000 & 1409 & 1370 & +30 & -00599 & $6 n+6) 1$ & $-(x)(0) 2$ \\
\hline 14,000 & 1108 & 1075 & +83 & .00618 & $0(t)=1$ & -010003 \\
\hline 15,000 & 801 & 752 & +40 & .00653 & $r 06610$ & -000018 \\
\hline 16,000 & 509 & 495 & +34 & .00724 & .00730 & -.00006 \\
\hline 17,000 & 297 & 296 & +1 & .00864 & .00866 & -.100002 \\
\hline 18,000 & 186 & 186 & 0 & .01065 & .01064 & +000001 \\
\hline
\end{tabular}

$\mathfrak{B}$. 
The representation of the numbers derived from the bars with pole-pieces affords results of a similar character. It is not necessary to present the figure showing the nature of the gain in accuracy by introduction of the term proportional to the magnetizing force, as it is precisely similar in character to that already given for plain bars. The two following Tables give the representation of these numbers in the same way as the two last. The coefficient of the magnetizing force in the second table is $12 \cdot 1$ nearly.

Bars with P.P. (pole-pieces) without Magnetizing-Force term.

$$
\mathrm{A}+\cdot 415(18366-\mathbf{B}) \cos \delta, \quad \begin{aligned}
& \log \frac{\kappa}{\rho}=2 \cdot 35933, \\
& \log _{f} f=\cdot 16500 .
\end{aligned}
$$

\begin{tabular}{|c|c|c|c|c|c|c|}
\hline \multirow{2}{*}{ 33. } & \multicolumn{2}{|c|}{$\mu$} & \multirow{2}{*}{ Diffs. } & \multicolumn{2}{|c|}{$\rho}$. & \multirow{2}{*}{ Diffs. } \\
\hline & Calc. & Obs. & & Calc. & Obs. & \\
\hline 0,000 & 290 & 293 & -3 & .00596 & $\cdot 00593$ & $+\cdot 00003$ \\
\hline 1,000 & 905 & 855 & +50 & 00362 & .00369 & -.00007 \\
\hline 2,000 & 1397 & 1250 & +147 & .00324 & .00332 & -.00008 \\
\hline 3,000 & 1772 & 1639 & +133 & .00308 & .00313 & -.00005 \\
\hline 4,000 & 2046 & 2041 & +5 & .00301 & .00301 & .00000 \\
\hline 5,000 & 2225 & 2222 & +3 & .00297 & .00297 & .00000 \\
\hline 6,000 & 2323 & 2381 & -58 & $\cdot 00295$ & $\cdot 00294$ & +.00001 \\
\hline 7,000 & 2352 & 2381 & -29 & $\cdot 00294$ & .00294 & .00000 \\
\hline 8,000 & 2323 & 2381 & -58 & .00295 & .00294 & $+\cdot 00001$ \\
\hline 9,000 & 2240 & 2326 & -86 & .00297 & .00295 & +.00002 \\
\hline 10,000 & 2115 & 2174 & -59 & 00299 & .00298 & $+\cdot 00001$ \\
\hline 11,000 & 1952 & 2041 & -89 & 00303 & .00301 & +00002 \\
\hline 12,000 & 1756 & 1754 & +2 & .00309 & .00309 & 00000 \\
\hline 13,000 & 1532 & 1408 & +124 & .00317 & .00323 & -00006 \\
\hline 14,000 & 1285 & 1031 & +254 & .00330 & .00349 & -.00019 \\
\hline 15,000 & 1051 & 595 & +456 & 00347 & .00420 & -00073 \\
\hline 16,000 & 733 & 300 & +433 & .00388 & .00585 & -00197 \\
\hline 17,000 & 432 & 207 & +225 & .00484 & .00734 & -.00250 \\
\hline 18,000 & 118 & 118 & 0 & .01100 & $\cdot 01103$ & -.00003 \\
\hline
\end{tabular}


Bars with P.P. (pole-pieces) with Magnetizing-Force term.

$$
\mu=\mathrm{A}=69\left(\frac{n \mathfrak{B}}{\mu}+\mathrm{B}-\mathbf{B}\right) \cos \delta, \quad \begin{array}{r}
\log \frac{\boldsymbol{\kappa}}{\rho}=2 \cdot 57346, \\
\log f=\cdot 16878 .
\end{array}
$$

\begin{tabular}{|c|c|c|c|c|c|c|}
\hline \multirow{2}{*}{33.} & \multicolumn{2}{|c|}{$\mu$. } & \multirow{2}{*}{ Diffs. } & \multicolumn{2}{|c|}{$\rho}$. & \multirow{2}{*}{ Diffs. } \\
\hline & Calc. & Obs. & & Galc. & Obs. & \\
\hline 0,000 & 297 & 293 & +4 & .00589 & .00593 & -00004 \\
\hline 1,000 & 894 & 855 & +39 & $\cdot 00364$ & .00369 & -.00005 \\
\hline 2,000 & 1384 & 1250 & +134 & $\cdot 00324$ & $\cdot 00332$ & -00008 \\
\hline 3,000 & 1767 & 1639 & +128 & .00309 & $\cdot 00313$ & -00004 \\
\hline 4,000 & 2056 & 2041 & +15 & .00301 & $\cdot 00301$ & $\cdot 00000$ \\
\hline 5,000 & 2250 & 2222 & +28 & $\cdot 00296$ & .00297 & -00001 \\
\hline 6,000 & 2358 & 2381 & -23 & $.00 \& 94$ & .00294 & .00000 \\
\hline 7,000 & 2389 & 2381 & +8 & .00234 & .00294 & .00000 \\
\hline 8,000 & 2351 & 2381 & -30 & .00294 & .00294 & $\cdot 00000$ \\
\hline 9,000 & 2249 & 2326 & -77 & .00296 & .00295 & +00001 \\
\hline 10,000 & 2091 & 2174 & -83 & .00300 & .00298 & +00002 \\
\hline 11,000 & 1882 & 2041 & -159 & $0030 \overline{5}$ & $\cdot 00301$ & +00004 \\
\hline 12,000 & 1627 & 1754 & -127 & .00313 & .00309 & +00004 \\
\hline 13,000 & 1339 & 1408 & -69 & .00327 & $\cdot 00323$ & +00004 \\
\hline 14,000 & 1021 & 1031 & -10 & 00350 & .00349 & +00001 \\
\hline 15,000 & 707 & 595 & +112 & .00393 & .00420 & -.00027 \\
\hline 16,000 & 451 & 300 & +151 & .00473 & .00585 & -.00112 \\
\hline 17,000 & 179 & 207 & -28 & .00810 & .00734 & +00076 \\
\hline 18,000 & 118 & 118 & 0 & 01102 & .01103 & -.00001 \\
\hline
\end{tabular}

Many rings have been completely examined in my various papers. It is a matter of interest to what extent these support the position that there is an absolute saturation limit, and to what extent they oppose it.

First, as to Rowland's own measures of the one ring which be examined completely. I have reduced them according to my formula at Phil. Mag. xix. p. 340. Although the magnetism is not carried very high, and the fitting has not been as perfectly accomplished as in my more recent calculations, yet it is clear that there is no indication of variation of the saturation limit. This generally shows itself in an excess of the calculated values of $\mu$ in the region $\mathbb{B}=15,000$ $-16,000$.

In all my own determinations the magnetism has beeñ carried up much higher; and I think in some of the rings discussed at Phil. Mag. xix. pp. 76-79 there is some appearance of this kind. But $I$ have repeatedly submitted nearly all of these to calculation, with the result that the introduction of the variable saturation limit, so as to fit the region in question, invariably 
unsettles the general representation of the values. So that in every case, without exception, the general representation of the behaviour of rings, furnished by calculation on the basis of a fixed saturation limit, has been far better than any obtained by using a variable saturation limit. At the same time it must be admitted that experiment sometimes shows a heightening of the apparent saturation limit as it is approached. The change, however, resembles rather a change in the properties of the metal than a continuation according to the same laws which successfully represent the general course of the values.

I must reserve for another occasion some considerations as to dynamo machines, founded on the numbers here obtained for bars with pole pieces.

It must be noticed that the bars here dealt with are only of one definite shape, viz. diameter $:$ length $:: 1: 20$ or thereabouts. And the object of the investigation was to get a series of reliable data with respect to this one shape, which might afford a sound starting-point for the investigation of other proportions.

\section{Intelligence and Miscellaneous Articles. \\ MEASUREMENT OF PITCH BY MANOMETRIC FLAMES. \\ BY M. DOUMER.}

W ANOMETRIC fiames have hitherto only been used as a means of demonstration, and for studying the quality of vowel sounds. They are, however, susceptible of more varied applications, and may in particular vie with the graphical method in studying the height of sounds.

For this purpose two adjacent manometric flames are taken, one of which is due to a sound of known height, the other to a sound the height of which is to be determined, and then by means of a rotating mirror it is ascertained how many vibrations of the sound under investigation correspond to a known number of vibrations of the chronograph. A simple proportion gives the desired height.

This method, which is very simple in theory, is in practice complicated, and almost impossible from the want of centering of the mirror. But it retains all its simplicity, and a certain elegance moreover, if we substitute a sensitive plate for the rotating mirror ; in other words, if the two manometric flames are simultaneously photograpked on the same plate.

The camera obscura used is a broad one, provided with a lens of 